\title{
Abbreviated National Early Warning Score predicts the need for hospital admission and in-hospital mortality in elderly patients
}

\author{
Toshiya Mitsunaga, ${ }^{1,2}$ Masahiko Hujita, ${ }^{2}$ Izumu Hasegawa, ${ }^{1}$ Kei Otani, ${ }^{1}$ Kenji Okuno, ${ }^{1}$ \\ Yuhei Ohtaki, ${ }^{1}$ Yutaka Seki, ${ }^{2}$ Kunihiro Mashiko, ${ }^{2}$ Satoshi Takeda ${ }^{1}$ \\ ${ }^{1}$ Department of Emergency Medicine, Jikei University School of Medicine; ${ }^{2}$ Department of Emergency \\ Medicine, Minamitama Hospital, Tokyo, Japan
}

\begin{abstract}
The aim of this study was to evaluate the value of the Abbreviated National Early Warning Score (aNEWS) for predicting admissions and in-hospital mortality in elderly patients present to Emergency Department (ED). This retrospective, single-centred observational study was carried out in the ED of Minamitama Hospital, in Tokyo, Japan from 1 April 2018 to 30 April 2018. All of the patients aged 65 and older were included in this study. The aNEWS is based on six common physiological vital signs, including peripheral oxygen saturation, the presence of inhaled oxygen parameters, body temperature, systolic blood pressure, pulse rate, and the Alert, responds to Voice, responds to Pain, Unresponsive score. The scores range from 0 and 3 for each parameter. The aNEWS ranged from a score of 0 to a maximum of 17 . The receiver operating characteristics (ROC) analysis was used to evaluate the predictive value of the aNEWS for admission and in-hospital mortality.

The median aNEWS of patients admitted to the hospital was significantly higher than that of patients discharged from the ED $(\mathrm{P}<0.001)$. The median aNEWS of survivors was significantly higher than that of non-survivors $(\mathrm{P}<0.001)$. The Areas under the
\end{abstract}

Correspondence: Toshiya Mitsunaga, Department of Emergency Medicine, Jikei University School of Medicine, 3-25-8 Nishi-Shinbashi, Minato-ku, Tokyo 105-8461, Japan.

Tel.: +81.42.663.0111 - Fax: +81.42.662.5890

E-mail: toshiya.m@jikei.ac.jp - toshi_promise_kt@yahoo.co.jp

Key words: National Early Warning Score; Elderly patients; Admission; Mortality; Risk score.

Contributions: TM, MH, IH, YS analysed the data; KOt, KOk, YO, KM, ST contributed to the design of the study; TM wrote the article. All authors contributed to the design and drafting the article.

Conflict of interest: the authors declare no potential conflict of interest.

Funding: none.

Received for publication: 15 August 2018.

Revision received: 16 October 2018.

Accepted for publication: 30 October 2018.

This work is licensed under a Creative Commons Attribution 4.0 License (by-nc 4.0).

CCopyright T. Mitsunaga et al., 2018

Licensee PAGEPress, Italy

Emergency Care Journal 2018; 14:7771

doi:10.4081/ecj.2018.7771
ROC Curve (AUC) for predicting admission was 0.773 [95\% CI 0.7142 to $0.8317, \mathrm{P}<0.001]$ for the aNEWS. The AUC for predicting in-hospital mortality was 0.791 [95\% CI 0.604 to 0.978 , $\mathrm{P}<0.001]$ for the aNEWS. Our single-centred study has demonstrated the utility of the aNEWS as a predictor of patient admission and in-hospital mortality in elderly patients.

\section{Introduction}

Life expectancy in Japan is the highest of all nations (83.7 years) because of improved health care. ${ }^{1}$ In Japan, the proportion of people older than 65 years was $23.0 \%$ in 2010 , and this is expected to reach $29.1 \%$ by 2020 , which is the highest in the world. ${ }^{2}$

As the life expectancy rises, the number of patients older than 65 years that present in Emergency Departments (EDs) is also increasing proportionately. A previous study published in the US reported that elderly patients that presented in EDs comprise about $50 \%$ of all ED patients. ${ }^{3}$

The increasing number of elderly patients presenting in EDs is one of the major causes of overcrowding in EDs. ${ }^{4-6}$ Moreover, elderly patients need specific management in the ED because of their unique physiological responses to acute disease. Furthermore, elderly patients normally stay in EDs longer than younger patients. ${ }^{7}$ Therefore, the decision whether to admit patients is an important factor in managing EDs.

Several risk-scoring systems have been developed to predict the risk of catastrophic deterioration and death of hospital inpatients. The National Early Warning Score (NEWS) was developed in 2012 in the UK by the National Early Warning Score Development and Implementation Group on behalf of the Royal College of Physicians. ${ }^{8}$

Previous studies have explored the association between ED NEWS with in-hospital mortality or ICU admission, and these findings suggested that this risk score also could be used as a triage tool to identify patients requiring admission to hospital. ${ }^{9-11}$ Several studies have reported the risk scoring systems for elderly patients who presented to ED, but they were not sufficiently effective for evaluating patients and none of them included calculations of NEWS. ${ }^{12-14}$

Because monitoring the respiratory rate is sometimes difficult, we proposed an Abbreviated National Early Warning Score (aNEWS) in order to make it easier to triage the patients and to increase the adherence of physicians.

The aim of this study was to evaluate the value of the aNEWS in predicting hospitalization and in-hospital mortality in patients older than 65 years who presented in the ED. 


\section{Materials and Methods}

\section{Design and setting}

This retrospective, single-centred observational study was conducted in the ED of Minamitama Hospital, an urban hospital in Tokyo, Japan. The hospital is a 122-beds facility providing general medicine, gastroenterological, cardiological, neurological, pulmonological, surgical, orthopaedic, urological, radiological, limited gynaecological, limited ophthalmological, and emergency medicine services to a population of approximately 560,000 people. This ED typically evaluates about 6,500 patients and accepts about 4,500 cases that present by ambulance annually.

\section{Study population}

This study was carried out between 1 April 2018 and 30 April 2018 at Minamitama Hospital, which is a secondary emergency medical institution. All of the patients aged 65 and older that presented to the ED for both surgical and non-surgical reasons during the study period were included in this study. Trauma patients were also included in this study but cardiopulmonary arrest (CPA) patients were excluded from this study.

\section{Data sources and measurements}

An emergency physician saw all the patients who presented in the ED, and he or she took over the patient's management and follow-up. During this process, all the data for each patient were recorded as electronic medical records by nurses. The patients were followed up until discharge, death or transfer to other hospitals. Data on each patient's discharge from the ED, admission to the hospital, and in-hospital mortality were recorded.

The aNEWS was calculated using the recorded physiological parameters for each patient. The aNEWS is derived from six common physiological vital signs, including peripheral oxygen saturation, the presence of oxygen supplementation, body temperature, systolic blood pressure, pulse rate, and the Alert, responds to Voice, responds to Pain, Unresponsive (AVPU) score. The scores range from 0 to 3 for each parameter. The aNEWS ranges from 0 to a maximum of 17 (Table 1). The AVPU was derived from the Glasgow Coma Scare as follows: $\mathrm{A}=14-15, \mathrm{~V}=9-13, \mathrm{P}=4-8, \mathrm{U}=3$.

Primary outcomes included hospitalization and in-hospital mortality. The patients were divided into two groups; Group 1 included those who were discharged from the ED. Group 2 included those who were admitted to the hospital. Patients who were transferred to another hospital were included in Group 2. To evaluate in-hospital mortality, the patients were divided into two groups: survivors and non-survivors.

\section{Statistical analysis}

The continuous variables were described as the medians and interquartile ranges, and they were compared using the Student's $t$ test and the Mann-Whitney $U$-test. The categorical variables were described as number (\%), and compared using the Pearson's $\chi^{2}$ test.

The receiver operating characteristics analysis was used to evaluate the predictive value of the aNEWS for admission and inhospital mortality. The cut-off points for the aNEWS were determined for both the primary outcomes using Youden's Index (sensitivity + specificity-1). Using these determined cut-off points, the sensitivity, and specificity of aNEWS were calculated for the admissions and the in-hospital mortality.

Data were analysed using SPSS (version 16.0; SPSS Inc., Chicago, Illinois, USA). A P value less than 0.05 was considered to indicate statistically significance.

\section{Results}

During the study period, 304 elderly patients presented to our ED. Forty-six cases were excluded because of their missing data, three cases were excluded because of CPA and finally 255 cases were ultimately included in this study. The median age (interquartile range) of the patients was $82(14.5)$ years, and $120(47.1 \%)$ of the patients were men. Seventy-five $(29.4 \%)$ patients arrived at the ED by ambulance. Fifty-six (22.0\%) gastroenterology cases, 43 (16.9\%) pulmonology cases, $38(14.9 \%)$ orthopaedic cases and 51 $(20.0 \%)$ miscellaneous cases were included, which accounted for $75 \%$ of all patients presenting in the ED. One-hundred thirty $(51.0 \%)$ patients were discharged from the ED, and $125(49.0 \%)$ patients were admitted including 17 cases that were transferred to another hospital. More than $75 \%$ of the patients did not need to receive oxygen, the consciousness of more than $75 \%$ of patients was clear, and fifty-six $(22.0 \%)$ were gastroenterology cases (Table 2).

The median aNEWS of patients admitted to the hospital was significantly higher than the median aNEWS of patients discharged from the ED $(\mathrm{P}<0.001)$. The systolic blood pressure of patients admitted to the hospital was significantly lower $(\mathrm{P}<0.05)$ and the temperature of patients admitted to the hospital was higher $(\mathrm{P}<0.001)$ than those of patients discharged from the ED. In the admission group, the percentage of patients, who received oxygen and whose state of consciousness was not obvious, was significantly higher than in the discharged group. On the other hand, there was no significant difference in age, sex ratio, pulse rate or oxygen saturation between the groups (Table 3 ). The in-hospital mortality rate was $3.1 \%$ ( 8 cases). The median aNEWS of survivors was significantly higher than the median aNEWS of non-survivors

Table 1. Abbreviated National Early Warning Score (aNEWS).

\begin{tabular}{lccccccc} 
& 3 & 2 & 1 & 0 & 1 & 2 \\
Systolic blood pressure (mmHg) & $\leq 90$ & $91-100$ & $101-110$ & $111-219$ & - & - & $\geq 220$ \\
Pulse rate $(\mathrm{bpm})$ & $\leq 40$ & - & $41-50$ & $51-90$ & $91-110$ & $111-130$ & $\geq 131$ \\
\hline Temperature $\left({ }^{\circ} \mathrm{C}\right)$ & $\leq 35.0$ & - & $35.1-36.0$ & $36.1-38.0$ & $38.1-39.0$ & $\geq 39.1$ & - \\
Oxygen saturation (\%) & $\leq 91$ & $92-93$ & $94-95$ & $\geq 96$ & - & - \\
\hline Inhaled oxygen & - & Yes & - & No & - & - \\
Alert, responds to Voice, responds to Pain, & - & - & - & A & - & - \\
Unresponsive (AVPU) & & & & & - \\
\hline
\end{tabular}

A, alert; $\mathrm{V}$, to voice; $\mathrm{P}$, to pain; $\mathrm{U}$, to unresponsive; bpm, beats or breaths per minute. 
$(\mathrm{P}<0.001)$. The pulse rate of survivors was significantly lower $(\mathrm{P}<0.05)$ than the pulse rate of non-survivors. In the non-survivors group, the percentage of patients, who received oxygen and whose state of consciousness was not obvious was significantly higher than those of the survivors group (Table 4).

The AUC for predicting admission was 0.773 [95\% confidence interval (CI) 0.7142 to $0.8317, \mathrm{P}<0.001]$ for aNEWS (Figure 1). The cut-off values for the aNEWS was 3 . The aNEWS of 3 or more had a sensitivity of $63.2 \%$, a specificity of $87.7 \%$, and an odds ratio of 12.24 for predicting admission. The AUC for predicting inhospital mortality was 0.791 [95\% confidence interval (CI) 0.604 to $0.978, \mathrm{P}<0.001$ ] for aNEWS (Figure 2). The cut-off value for the aNEWS was 3 . The aNEWS of 3 or more had a sensitivity of 87.5 $\%$, a specificity of $64.4 \%$, and an odds ratio of 12.65 for predicting in-hospital mortality.

\section{Discussion}

In this study, we demonstrated that the aNEWS has potential as triage tool that can be used to predict the admission rates of elderly patients presenting in EDs. In several studies, the effectiveness of

Table 2. Baseline characteristics of the study population.

\begin{tabular}{|c|c|}
\hline & $\begin{array}{c}\text { Median (interquartile range) } \\
(\mathrm{n}=255)\end{array}$ \\
\hline Age, years & $82(14.5)$ \\
\hline $\begin{array}{l}\text { Sex }[n(\%)] \\
\text { Male } \\
\text { Female }\end{array}$ & $\begin{array}{l}120(47.1) \\
135(52.9)\end{array}$ \\
\hline $\begin{array}{l}\text { Diagnostic Categoly [n (\%)] } \\
\text { Cardiology } \\
\text { Pulmonology } \\
\text { Neurology } \\
\text { Gatroenterology } \\
\text { Diabetes } \\
\text { Urology } \\
\text { Orthopedic } \\
\text { Others }\end{array}$ & $\begin{array}{c}29(11.4) \\
43(16.9) \\
16(6.3) \\
56(22.0) \\
3(1.2) \\
19(7.5) \\
38(14.9) \\
51(20.0)\end{array}$ \\
\hline $\begin{array}{l}\text { Route of presentation [n (\%)] } \\
\text { Walk in } \\
\text { Ambulance }\end{array}$ & $\begin{array}{l}180(70.6) \\
75(29.4)\end{array}$ \\
\hline $\begin{array}{l}\text { Disposition [n (\%)] } \\
\text { Discharge } \\
\text { Admission }\end{array}$ & $\begin{array}{l}130(51.0) \\
125(49.0)\end{array}$ \\
\hline Systolic blood pressure (mmHg) & $142(35.5)$ \\
\hline Pulse rate (bpm) & $82(23)$ \\
\hline Temperature $\left({ }^{\circ} \mathrm{C}\right)$ & $36.7(0.8)$ \\
\hline Oxygen saturation (\%) & $97(3)$ \\
\hline $\begin{array}{l}\text { Inhaled oxygen [n (\%)] } \\
\text { Yes } \\
\text { No }\end{array}$ & $\begin{array}{c}63(24.7) \\
192(75.3)\end{array}$ \\
\hline $\begin{array}{l}\text { AVPU }[\mathrm{n}(\%)] \\
\quad \text { A } \\
\quad \text { Non-A }\end{array}$ & $\begin{array}{c}197(77.3) \\
58(22.7)\end{array}$ \\
\hline aNEWS & $1(4)$ \\
\hline
\end{tabular}

Data are presented as the median (interquartile range) for continuous variables and the number (\%) for categorical variables. aNEWS, Abbreviated National Early Warning Score; bpm, beats or breaths per minute. the NEWS for predicting admissions to a critical care unit has been evaluated, but there have been no studies evaluating the effectiveness of the NEWS for predicting admissions to hospitals or EDs. One study was carried out by Zerrin Defne et al., ${ }^{15}$ which examined the predictive value of the VitalPac Early Warning Score (ViEWS). ViEWS is the basis of the NEWS, and it uses seven of the same vital signs, for admission, which were found to be moderate, yielding an AUC of 0.756 . In contrast, the study carried out by Murray et al. ${ }^{16}$ demonstrated that the abbreviated VIEWS (in which mental state was excluded) is insufficient to predict the decision regarding hospital admission. In our study, we found that the aNEWS is moderately effective for predicting admission in elderly patients, and the AUC for admission was 0.773.

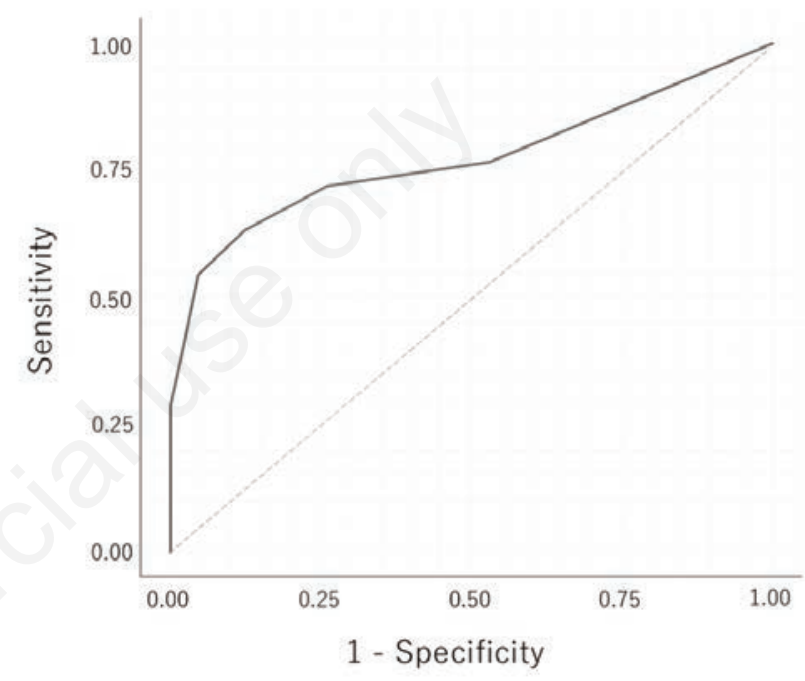

Figure 1. Receiver operator characteristics curves for the Abbreviated National Early Warning Score for admission.

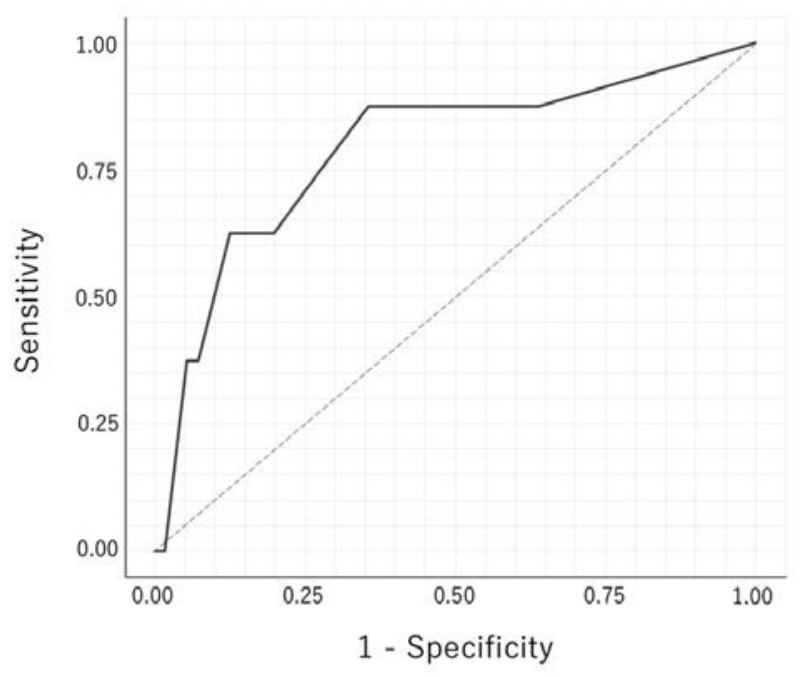

Figure 2. Receiver operator characteristics curves for the Abbreviated National Early Warning Score for in-hospital mortality. 
In this study, we also showed that the aNEWS is an effective triage tool that can be used to predict the in-hospital mortality in elderly patients presenting at an ED. In the study carried out by Smith et al., ${ }^{17}$ the predictive value of the NEWS for in-hospital mortality was found to be high, yielding an AUC of 0.894. In the study carried out by Kovacs et al., ${ }^{18}$ the predictive value of the NEWS for predicting in-hospital mortality of different groups showed similar results. In the study carried out by Leandro et al., ${ }^{19}$ the predictive value of the two kinds of short NEWS (which did not include temperature as a factor or temperature and systolic blood pressure) for predicting a patient's deterioration was extremely high, yielding AUCs of 0.965 and 0.903 , respectively. Although several studies of the NEWS for in-hospital mortality were carried out, none of them were specific for elderly patients.
In our study, the predictive value of the aNEWS for in-hospital mortality in elderly patients was found to be moderate. The AUC for in-hospital mortality was 0.791 . In a previous study carried out by Abbott et al., ${ }^{20}$ the mortality rate was $4.7 \%$ (15 patients out of 322). In our study, the in-hospital mortality rate was $3.1 \%$, which is lower than this previous study.

This study has several limitations. Compared to previous studies, the cut-off values for the aNEWS in admission and in-hospital mortality were low in our study, and the condition of patients presenting in our ED was not severe. First, it was expected that the reason for this was that elderly patients were admitted not because of their physical severity, but because of social problems, such as a lack of families who care about the patients. Secondly, the patient's vital signs are often unchanged on presentation with prob-

Table 3. Comparison of parameters between the discharged group and the admitted group.

\begin{tabular}{|c|c|c|c|}
\hline & $\begin{array}{l}\text { Median (in } \\
\text { Group } 1 \\
\text { (discharged from ED) } \\
(\mathrm{n}=130)\end{array}$ & $\begin{array}{l}\text { tile range) } \\
\text { Group } 2 \\
\text { (Admission to the hospital) } \\
\text { ( } \mathrm{n}=125)\end{array}$ & $P$ value \\
\hline Age, years & $81(15)$ & $82(14)$ & 0.27 \\
\hline $\begin{array}{c}\text { Sex }[\mathrm{n}(\%)] \\
\text { Male } \\
\text { Female } \\
\end{array}$ & $\begin{array}{l}59(45.4) \\
71(54.6)\end{array}$ & $\begin{array}{l}61(48.8) \\
64(51.2)\end{array}$ & 0.585 \\
\hline Systolic blood pressure (mmHg) & $148(37.75)$ & $137(34)$ & $<0.05$ \\
\hline Pulse rate (bpm) & $81.5(25)$ & $84(22)$ & 0.156 \\
\hline Temperature $\left({ }^{\circ} \mathrm{C}\right)$ & $36.6(0.6)$ & $37(1.1)$ & $<0.001$ \\
\hline Oxygen saturation (\%) & $97(2)$ & $97(4)$ & 0.258 \\
\hline $\begin{array}{l}\text { Inhaled oxygen }[\mathrm{n}(\%)] \\
\text { Yes } \\
\text { No }\end{array}$ & $\begin{array}{c}1(0.8) \\
129(99.2)\end{array}$ & $\begin{array}{l}62(49.6) \\
63(50.4)\end{array}$ & $<0.001$ \\
\hline $\begin{array}{l}\text { AVPU }[n(\%)] \\
\quad \text { A } \\
\text { Non-A }\end{array}$ & $\begin{array}{c}126(96.9) \\
4(3.1)\end{array}$ & $\begin{array}{l}71(56.8) \\
54(43.2)\end{array}$ & $<0.001$ \\
\hline aNEWS & $1(2)$ & $4(5)$ & $<0.001$ \\
\hline
\end{tabular}

Data are presented as the median (interquartile range) for continuous variables and the number (\%) for categorical variables. aNEWS, Abbreviated National Early Warning Score; bpm, beats or breaths per minute.

Table 4. Comparison of parameters between survivors and non-survivors.

\begin{tabular}{|c|c|c|c|}
\hline & $\begin{array}{l}\text { Survivors } \\
(\mathrm{n}=247)\end{array}$ & $\begin{array}{c}\text { Non-survivors } \\
(\mathrm{n}=8)\end{array}$ & P value \\
\hline Age, years & $82(14.5)$ & $87.5(12)$ & 0.398 \\
\hline $\begin{array}{c}\text { Sex }[\mathrm{n}(\%)] \\
\text { Male } \\
\text { Female } \\
\end{array}$ & $\begin{array}{l}116(46.96) \\
131(53.04)\end{array}$ & $\begin{array}{l}4(50.00) \\
4(50.00)\end{array}$ & 0.866 \\
\hline Systolic blood pressure (mmHg) & $143(35.5)$ & $130(37)$ & 0.423 \\
\hline Pulse rate (bpm) & $82(23.5)$ & $90.5(25)$ & $<0.05$ \\
\hline Temperature $\left({ }^{\circ} \mathrm{C}\right)$ & $36.7(0.8)$ & $36.95(0.725)$ & 0.251 \\
\hline Oxygen saturation (\%) & $97(3)$ & $98.5(4.25)$ & 0.67 \\
\hline $\begin{array}{l}\text { Inhaled oxygen [n (\%)] } \\
\text { Yes } \\
\text { No }\end{array}$ & $\begin{array}{c}58(23.5) \\
189(76.5)\end{array}$ & $\begin{array}{l}5(62.5) \\
3(37.5)\end{array}$ & $<0.05$ \\
\hline $\begin{array}{l}\text { AVPU [n (\%)] } \\
\quad \mathrm{A} \\
\quad \text { Non-A }\end{array}$ & $\begin{array}{c}195(79.0) \\
52(21.0)\end{array}$ & $\begin{array}{l}2(25.0) \\
6(75.0)\end{array}$ & $<0.001$ \\
\hline aNEWS & $1(4)$ & $6(4.25)$ & $<0.001$ \\
\hline
\end{tabular}

Data are presented as the median (interquartile range) for continuous variables and the number (\%) for categorical variables. aNEWS, Abbreviated National Early Warning Score; bpm, beats or breaths per minute. 
lems such as limbs fracture, peripheral vertigo and intoxication. Because of the low severity of their physical illness, the number of non-survivors was very few. Therefore, several biases, such as the increase in false-negatives, would be statistically likely to occur. In our hospital, we lack several departments, such as the Resuscitation Unit, Neurosurgery, Cardiac Surgery and Trauma Surgery Department. Thus, we have to consider the selection bias during the pre-hospital phase. We researched the data for elderly patients presenting in the ED in April only, so there were seasonal variations and longer-term study will be needed to examine seasonal biases. Because ED physicians change daily, criteria for admission were different. Therefore, further study of a longer duration that includes larger numbers of patients will be needed.

\section{Conclusions}

Our single-centred study has demonstrated the utility of the aNEWS as a predictor of patient admission and in-hospital mortality in elderly patients. Further studies are required to evaluate the impact of aNEWS on more critically ill patients who come to the resuscitation centre. These studies should likewise evaluate whether aNEWS is useful in other geographical areas, including rural regions. Further studies are also needed to show whether aNEWS can be used to help manage the length of stay in EDs and, therefore, reduce ED crowding.

\section{References}

1. World Health Organization (WHO). World Health Statistics 2017: Monitoring health for the SDGs. 2017. Available from: https://www.who.int/gho/publications/world_health_statistics/2017/en/

2. Sbiti-Rohr D, Kutz A, Christ-Crain M, et al. The National Early Warning Score (NEWS) for outcome prediction in emergency department patients with community-acquired pneumonia: results from a 6-year prospective cohort study. BMJ Open 2016;6:e011021.

3. Abbott TEF, Cron N, Vaid N, et al. Pre-hospital National Early Warning Score (NEWS) is associated with in-hospital mortality and critical care unit admission: A cohort study. Ann Med Surg 2018;27:17-21.

4. Emergency Promotion Foundation Survey Research Grant Project. Prospective study on the reality of emergency transportation acceptance refusal. 2009.

5. Ministry of Health, Labor and Welfare. The way of emergency medical system for elderly patients. Japan: Ministry of Health; 2013.

6. Ministry of Internal Affairs and Communications Fire and Disaster Management Agency. Current status of emergency and rescue in 2016. Japan: Ministry of Internal Affairs and Welfare; 2016.

7. Roberts DC, Mckay MP, Shaffer A. Increasing rates of emer- gency department visits for elderly patients in the United States, 1993 to 2003. Ann Emerg Med 2008;51:769-74.

8. Royal College of Physicians. National Early Warning Score (NEWS): Standardising the assessment of acute-illness severity in the NHS. Report of working party. London, UK: Royal College of Physicians; 2012. Available from: https://www.rcplondon.ac.uk/projects/outputs/national-earlywarning-score-news-2

9. Subbe CP, Kruger M, Rutherford P, Gemmel L. Validation of a modified Early Warning Score in medical admissions. QJM 2011;10:521-6.

10. Burch VC, Tarr G, Morroni C. Modified early warning score predicts the need for hospital admission and in hospital mortality. Emerg Med J 2008;10:674-8.

11. Cei M, Bartolomei C, Mumoli N. In-hospital mortality and morbidity of elderly medical patients can be predicted at admission by the Modified Early Warning Score: A prospective study. Int J Clin Pract 2009;4:591-5.

12. Subbe CP, Davies RG, Williams E, et al. Effect of introducing the Modified Early Warning score on clinical outcomes, cardiopulmonary arrests and intensive care utilization in acute medical admissions. Anaesthesia 2003;58:797-802.

13. Salvi F, Morichi V, Grilli A, et al. Predictive validity of the Identification of Seniors At Risk (ISAR) screening tool in elderly patients presenting to two Italian Emergency Departments. Aging Clin Exp Res 2009;21:69-75.

14. Buurman BM, van den Berg W, Korevaar JC, et al. Risk for poor outcomes in older patients discharged from an emergency department: Feasibility of four screening instruments. Eur J Emerg Med 2011;18:215-20.

15. Dundar ZD, Ergin M, Karamercan MA, et al. Modified Early Warning Score and VitalPac Early Warning Score in geriatric patients admitted to emergency department. Eur J Emrg Med 2016;23:406-12.

16. Murray A, Kellett J, Huang W, et al. Trajectories of the averaged abbreviated Vitalpac early waring score (AbEWS) and clinical course of 44,531 consecutive admissions hospitalized for acute medical illness. Resuscitation 2014;85:544-8.

17. Smith GB, Prytherch DR, Meredith P, et al. The ability of the National Early Warning Score (NEWS) to discriminate patients at risk of early cardiac arrest, unanticipated intensive care unit admission, and death. Resuscitation 2013;84:465-70.

18. Kovacs C, Jarvis SW, Prytherch DR, et al. Comparison of the National Early Earning Score in non-elective medical and surgical patients. Br J Surg 2016;103:1385-93.

19. Luís L, Nunes C. Short National Early Warning Score Developing a Modified Early Warning Score. Aust Crit Care 2018;31:376-81.

20. Abbott TEF, Torrance HDT, Cron N, et al. A single-centre cohort study of National Early Warning Score (NEWS) and near patient testing in acute medical admissions. Eur J Int Med 2016;35:78-82. 